\title{
A topological view on L-fuzzy soft sets: Degree of countably compactness and the Lindelöf property
}

\author{
Vildan Çetkin \\ Department of Mathematics, Kocaeli University, Umuttepe Campus, Kocaeli, Turkey
}

Received: 2 May 2020, Accepted: 5 March 2021

Published online: 21 March 2021.

\begin{abstract}
In the present article, the parameterized degree of countably compactness and having the Lindelöf property of a lattice valued fuzzy soft set, are pictured in terms of implication operation of the underlying lattice. Many fundamental features of countably compactness and the Lindelöf property are observed in the context of lattice valued fuzzy soft sets by regarding the parametrization tool. It is also shown that an $L$-fuzzy soft set having the Lindelöf property is countably compact if and only if it is compact in the described manner.
\end{abstract}

Keywords: Fuzzy soft set, fuzzy soft topology, compactness, countably compactness, Lindelöf property.

\section{Introduction}

The soft set theory, initiated by Molodtsov [24] in 1999, is one of the mathematical methods that aims to describe phenomena and concepts of ambiguous, undefined and imprecise meaning. The fuzzy soft set theory which is obtained by including softness to the fuzzy sets defined by Maji et al. [23], opened a new perspective for researchers working on diverse areas. Nowadays, it is not surprised to see papers on both of theoretics and applications inspired by the soft set and the fuzzy soft set theory $[1,2,3,8,13,22,25,26,33,34]$.

As we all know in general topology, the notion of compactness is sort of a topological generalization of finiteness. The notion of compactness has been applied to crisp-fuzzy case by Chang [10] by means of open covers. Afterward, many researchers have successfully generalized the compactness theory of general topology to fuzzy setting in different aspects [20,21,29,30,32]. The compactness theory has been extended to the fuzzy soft universe by Çetkin et al. [12]. Later in her seminal papers Çetkin $[14,15]$ have presented the parameterized degree of semi-precompactness and the compactness in the fuzzy soft universe. The definitions of countable compactness and the Lindelöf property in $L$-topological spaces have been introduced by Shi [31]. Besides we refer $[4,5,6,16,17]$ for the compactness in the soft unverse.

This paper aims to describe the parameterized degree of fuzzy countably compactness and the Lindelöf property as a continuation of the studies related with parameterized degree of compactness [14,15]. In this manner the article is arranged as follows. In section 2, we recall some lattice theoretical definitions and basic notions and notations necessary for the whole paper. In section 3, we present the notion of countably compactness degree of an $L$-fuzzy soft set and discuss the elementary features. In the last section, we picture the notion of Lindelöfness degree of an $L$-fuzzy soft set with its properties. In conclude, we investigate the relations among parameterized compactness degree, countably compactness degree and the degree of having the Lindelöf property. 


\section{Preliminaries}

Throughout this paper, $X$ refers to a nonempty initial universe, $E, K$ denotes the arbitrary nonempty sets viewed on the sets of parameters and $L=\left(L, \vee, \wedge,^{\prime}\right)$ denotes a complete DeMorgan algebra with the smallest element $0_{L}$ and the largest element $1_{L}$. With the underlying lattice $L$, a mapping $A: X \rightarrow L$ is said to be an $L$-fuzzy set on $X$ and by $L^{X}$, we denote the family of all $L$-fuzzy sets on $X$.

Let $\alpha, \beta$ and $\gamma$ be elements in $L$. An element $\alpha$ in $L$ is said to be coprime if $\alpha \leq \beta \vee \gamma$ implies that $\alpha \leq \beta$ or $\alpha \leq \gamma$. The set of all coprime elements of $L$ is denoted by $c(L)$. We say $\alpha$ is wedge below $\beta$, in symbols, $\alpha \triangleleft \beta$ or $\beta \triangleright \alpha$, if for every arbitrary subset $D \subseteq L, \vee D \geq \beta$ implies $\alpha \leq d$ for some $d \in D$. As shown by Raney [27] a complete lattice $L$ is completely distributive if and only if $\beta=\bigvee\{\alpha \in L \mid \alpha \triangleleft \beta\}$ for each $\beta \in L$. The wedge below operation in a completely distributive lattice has an interpolation property, this means $\alpha \triangleleft \beta$ implies there exists $\gamma \in L$ such that $\alpha \triangleleft \gamma \triangleleft \beta$. For the details of lattices, see [18].

The binary operation $\mapsto$ in the complete DeMorgan algebra $L$ is given by $\alpha \mapsto \beta=\bigvee\{\gamma \in L \mid \alpha \wedge \gamma \leq \beta\}$. For all $\alpha, \beta, \gamma, \delta \in L$ and $\left\{\alpha_{i}\right\},\left\{\beta_{i}\right\} \subseteq L$, the following properties are satisfied:

(1) $(\alpha \mapsto \beta) \geq \gamma$ iff $\alpha \wedge \gamma \leq \beta$.

(2) $\alpha \mapsto \beta=1_{L}$ iff $\alpha \leq \beta$.

(3) $\alpha \mapsto\left(\bigwedge_{i} \beta_{i}\right)=\bigwedge_{i}\left(\alpha \mapsto \beta_{i}\right)$.

(4) $\left(\bigvee_{i} \alpha_{i}\right) \mapsto \beta=\bigwedge_{i}\left(\alpha_{i} \mapsto \beta\right)$.

The parameterized version of an $L$-fuzzy set is called an $L$-fuzzy soft set and it is defined as follows.

Definition 1. [23] $f$ is called an $L$-fuzzy soft set on $X$, where $f$ is a mapping from $E$ into $L^{X}$. This means that $f_{e}:=f(e): X \rightarrow L$, is an $L$-fuzzy set on $X$, for each parameter $e \in E$.

The family of all $L$-fuzzy soft sets on $X$ is denoted by $\left(L^{X}\right)^{E}$.

From now on, for a subfamily $\mathscr{U} \subseteq\left(L^{X}\right)^{E}$, the symbols $2^{(\mathscr{U})}$ and $2^{|\mathscr{U}|}$ will demonstrate the set of all finite subfamilies and countable subfamilies of $\mathscr{U}$, respectively.

Definition 2. [7,23,28] Let $f$ and $g$ be two $L$-fuzzy soft sets on $X$, then

(1) We say that $f$ is an $L$-fuzzy soft subset of $g$ and write $f \sqsubseteq g$ if $f_{e} \leq g_{e}$, for each $e \in E$. $f$ and $g$ are called equal if $f \sqsubseteq g$ and $g \sqsubseteq f$.

(2) The union of $f$ and $g$ is an $L$-fuzzy soft set $h=f \sqcup g$, where $h_{e}=f_{e} \vee g_{e}$, for each $e \in E$.

(3) The intersection of $f$ and $g$ on $X$ is an $L$-fuzzy soft set $h=f \sqcap g$, where $h_{e}=f_{e} \wedge g_{e}$, for each $e \in E$.

(4) The complement of an $L$-fuzzy soft set $f$ is denoted by $f^{\prime}$, where $f^{\prime}: E \rightarrow L^{X}$ is a mapping given by $f_{e}^{\prime}=\left(f_{e}\right)^{\prime}$, for each $e \in E$. Clearly $\left(f^{\prime}\right)^{\prime}=f$.

Definition 3. [28]

(1) An $L$-fuzzy soft set $f$ on $X$ is called a null $L$-fuzzy soft set and denoted by $\widetilde{0}$, if $f_{e}(x)=0$, for each $e \in E$ and $x \in X$.

(2) An $L$-fuzzy soft set $f$ on $X$ is called an absolute $L$-fuzzy soft set and denoted by $\widetilde{1}$, if $f_{e}(x)=1$, for each $e \in E, x \in X$. Clearly $(\widetilde{1})^{\prime}=\widetilde{0}$ and $\widetilde{0}^{\prime}=\widetilde{1}$.

Definition 4. [14] The fuzzy soft inclusion $[\widetilde{\sqsubseteq}]:\left(L^{X}\right)^{E} \times\left(L^{X}\right)^{E} \rightarrow L$ is defined by the following equality

$$
[f \widetilde{\complement} g]=\bigwedge_{x \in X} \bigwedge_{e \in E}\left(f_{e}^{\prime}(x) \vee g_{e}(x)\right) .
$$


Definition 5. [19] Let $\varphi: X_{1} \rightarrow X_{2}$ and $\psi: E_{1} \rightarrow E_{2}$ be two functions, where $E_{1}$ and $E_{2}$ are parameter sets for the crisp sets $X_{1}$ and $X_{2}$, respectively. Then the pair $\varphi_{\psi}$ is called an $L$-fuzzy soft mapping from $X_{1}$ to $X_{2}$. Let $f$ and $g$ be two $L$-fuzzy soft sets on $X_{1}$ and $X_{2}$, respectively.

(1) The image of $f$ under the $L$-fuzzy soft mapping $\varphi_{\psi}$, denoted by $\varphi_{\psi}(f)$, is an $L$-fuzzy soft set on $X_{2}$ defined by for all $k \in E_{2}, y \in X_{2}$,

$$
\varphi_{\psi}(f)_{k}(y)= \begin{cases}\bigvee \bigvee_{\varphi(x)=y} \bigvee_{\psi(a)=k} f_{a}(x), & \text { if } x \in \varphi^{-1}(y), a \in \psi^{-1}(k), \\ 0, & \text { otherwise. }\end{cases}
$$

(2) The pre-image of $g$ under the $L$-fuzzy soft mapping $\varphi_{\psi}$, denoted by $\varphi_{\psi}^{-1}(g)$, is an $L$-fuzzy soft set on $X_{1}$ defined by $\varphi_{\psi}^{-1}(g)_{e}(x)=g_{\psi(e)}(\varphi(x))$, for all $e \in E_{1}, x \in X_{1}$. If $\varphi$ and $\psi$ is injective (surjective), then $\varphi_{\psi}$ is said to be injective (surjective).

Definition 6. [11] A parameterized family $\mathscr{T}=\left\{\mathscr{T}_{k}\right\}_{k \in K}$ of $\mathscr{T}_{k} \subseteq\left(L^{X}\right)^{E}$ which satisfies the following properties for each $k \in K$ is called an $(E, K)$-soft $L$-topology on $X$ :

(S1) $\widetilde{0}, \widetilde{1} \in \mathscr{T}_{k}$.

(S2) If $f, g \in \mathscr{T}_{k}$, then $f \sqcap g \in \mathscr{T}_{k}$.

(S3) If $\left\{f_{i}\right\}_{i \in \Gamma} \subseteq \mathscr{T}_{k}$, then $\bigsqcup_{i \in \Gamma} f_{i} \in \mathscr{T}_{k}$.

The pair $(X, \mathscr{T})$ is called an $(E, K)$-soft $L$-topological space. $f$ is called open according to $k \in K$ if $f \in \mathscr{T}_{k}$, and it is called closed according to $k \in K$ if $f^{\prime} \in \mathscr{T}_{k}$

Definition 7. [9] A mapping $\tau: K \rightarrow L^{\left(L^{X}\right)^{E}}$ is called an $L$-fuzzy $(E, K)$-soft topology on $X$ if it satisfies the following conditions for each $k \in K$.

(O1) $\tau_{k}(\widetilde{0})=\tau_{k}(\widetilde{1})=1_{L}$.

(O2) $\tau_{k}(f \sqcap g) \geq \tau_{k}(f) \wedge \tau_{k}(g)$, for all $f, g \in\left(L^{X}\right)^{E}$.

(O3) $\tau_{k}\left(\bigsqcup_{i \in \Delta} f_{i}\right) \geq \bigwedge_{i \in \Delta} \tau_{k}\left(f_{i}\right)$, for all $f_{i} \in\left(L^{X}\right)^{E}, i \in \Delta$.

Then the pair $(X, \tau)$ is called an $L$-fuzzy $(E, K)$-soft topological space. The value $\tau_{k}(f)$ is interpreted as the degree of openness of an $L$-fuzzy soft set $f$ with respect to the parameter $k \in K$. The parameterized gradation of closedness of $f$ is computed as $\tau_{k}^{*}(f)=\tau_{k}\left(f^{\prime}\right)$, where $f^{\prime}$ denotes the complement of the $L$-fuzzy soft set $f$.

Let $\mathscr{U}$ be a subfamily of $\left(L^{X}\right)^{E}$, then the value $\tau_{k}(\mathscr{U})=\bigwedge_{f \in \mathscr{U}} \tau_{k}(f)$ will be called the parameterized openness degree of the subfamily $\mathscr{U} \subseteq\left(L^{X}\right)^{E}$ with respect to the parameter $k \in K$.

Remark. [14] It is clear that if $\mathscr{T}=\left\{\mathscr{T}_{k}\right\}_{k \in K}$ is an $(E, K)$-soft $L$-topology on $X$, then the mapping $\tau: K \rightarrow L^{\left(L^{X}\right)^{E}}$ defined by $\tau(k):=\tau_{k}=\chi_{\mathscr{T}_{k}}$ is an $(E, K)$-soft $L$-topology on $X$, where

$$
\chi_{T_{k}}(h)= \begin{cases}1_{L}, & \text { if } h \in \mathscr{T}_{k}, \\ 0_{L}, & \text { if } h \notin \mathscr{T}_{k}\end{cases}
$$

Example 1. [12] Let $L=\{(0,0),(1,1)\} \cup\{(a, 0),(0, b),(a, a) \mid a, b \in(0,1)\} . " \leq$ " is defined as follows: $(m, b) \leq(n, d)$ if and only if $m \leq n$ and $b \leq d$. Define an order reversing involution ' $: L \rightarrow L$ is as follows: for each $x, y \in(0,1)$, $(x, 0)^{\prime}=(1-x, 0),(0, y)^{\prime}=(0,1-y),(x, x)^{\prime}=(1-x, 1-x)$ and $(1,1)^{\prime}=(0,0)$. Then $\left(L, \leq,^{\prime}\right)$ is a complete DeMorgan algebra. Let $X=\{x, y\}, E=(0,0.5]$ and $f_{e}(x)=f_{e}(y)=(e, 0), g_{e}(x)=g_{e}(y)=(0, e)$ and $h_{e}(x)=h_{e}(y)=(e, e)$ for each $e \in E$. Define a mapping $\tau: E \rightarrow L^{\left(L^{X}\right)^{E}}$ as follows: 


$$
\tau_{e}(u)= \begin{cases}(1,1), & \text { if } u \in\{\widetilde{0}, \widetilde{1}, h\} \\ (e, 0), & \text { if } u=f \\ (0, e), & \text { if } u=g \\ (0,0), & \text { otherwise }\end{cases}
$$

Then $\tau$ is an $L$-fuzzy $(E, E)$-soft topology on $X$.

Definition 8. [9] Let $\left(X_{1}, \tau^{1}\right)$ be an $L$-fuzzy $\left(E_{1}, K_{1}\right)$-soft topological space and $\left(X_{2}, \tau^{2}\right)$ be an $L$-fuzzy $\left(E_{2}, K_{2}\right)$-soft topological space. Let $\varphi: X_{1} \rightarrow X_{2}, \psi: E_{1} \rightarrow E_{2}$ and $\eta: K_{1} \rightarrow K_{2}$ be functions. Then $\varphi_{\psi, \eta}:\left(X_{1}, \tau^{1}\right) \rightarrow\left(X_{2}, \tau^{2}\right)$ is said to be continuous if $\tau_{k}^{1}\left(\varphi_{\psi}^{-1}(g)\right) \geq \tau_{\eta(k)}^{2}(g)$ for all $g \in\left(L^{X_{2}}\right)^{E_{2}}, k \in K_{1}$.

Lemma 1. [12] Let $\varphi: X_{1} \rightarrow X_{2}, \psi: E_{1} \rightarrow E_{2}$ and $\eta: K_{1} \rightarrow K_{2}$ be three crisp functions. Then for each subfamily $\mathscr{U} \subseteq$ $\left(L^{X_{2}}\right)^{E_{2}}$, the following equality is satisfied.

$$
\bigvee_{k \in E_{2}} \bigvee_{y \in X_{2}}\left(\varphi_{\psi}(g)_{k}^{\prime}(y) \wedge \bigwedge_{f \in \mathscr{U}} f_{k}(y)\right)=\bigvee_{e \in E_{1}} \bigvee_{x \in X_{1}}\left(g_{e}^{\prime}(x) \wedge \bigwedge_{f \in \mathscr{U}} \varphi_{\psi}^{-1}(f)_{e}(x)\right)
$$

Definition 9. [15] Let $/ X, \tau)$ be an $L$-fuzzy $(E, K)$-soft topological space and $g \in\left(L^{X}\right)^{E}$. Then the compactness degree of $g$ with respect to the parameter $k$ is defined as follows.

$$
\operatorname{com}_{\tau}(k, g)=\bigwedge_{\mathscr{U} \subseteq\left(L^{X}\right)^{E}}\left(\tau_{k}(\mathscr{U}) \mapsto\left([g \widetilde{\sqsubseteq} \bigvee \mathscr{U}] \mapsto \bigvee_{\mathscr{V} \in 2^{(\mathscr{U})}}[g \widetilde{\subseteq} \bigvee \mathscr{V}]\right)\right)
$$

\section{Parameterized Degree of Countably Compactness}

In order to generalize the notion of countably compactness to the fuzzy soft universe by means of fuzzy-fuzzy case, let us consider the following definition which gives us the parameterized version of the fuzzy-crisp case. Throughout this study, the collection of all countable subfamilies of $\left(L^{X}\right)^{E}$ will be denoted by the symbol $\mathscr{C}\left(L^{X}\right)^{E}$.

Definition 10. Let $\mathscr{T}=\left\{\mathscr{T}_{k}\right\}_{k \in K}$ be an $(E, K)$-soft L-topology on $X$ and $h \in\left(L^{X}\right)^{E}$. The L-fuzzy soft set $h$ is said to be countably compact in $(X, \mathscr{T})$, if for each $k$ and each countable open $k$-cover $\mathscr{U} \subseteq \mathscr{T}_{k}$ of $h$ there exists a finite subfamily $\mathscr{V} \subseteq \mathscr{U}$ which covers $h$, i.e.,

$$
[h \widetilde{\Xi} \bigvee \mathscr{U}] \leq \bigvee_{\mathscr{V} \in 2^{(\mathscr{U})}}[h \tilde{\Xi} \bigvee \mathscr{V}]
$$

This means that

$$
[h \widetilde{\Xi} \bigvee \mathscr{U}] \leq \bigvee_{\mathscr{V} \in 2^{(\mathscr{U})}}[h \tilde{\subseteq} \bigvee \mathscr{V}] \Leftrightarrow\left[[h \tilde{\Xi} \bigvee \mathscr{U}] \leq \bigvee_{\mathscr{V} \in 2^{(\mathscr{U})}}[h \widetilde{\Xi} \bigvee \mathscr{V}]\right]=1_{L}
$$

For each $k \in K, \mathscr{U} \subseteq \mathscr{T}_{k}$ countable, we define $\chi_{\mathscr{T}_{k}}(\mathscr{U})=1_{L}$, where $\chi_{\mathscr{T}_{k}}(\mathscr{U})=\bigwedge_{f \in \mathscr{U}} \chi_{\mathscr{T}_{k}}(f)$. Hence we may infer that $h \in\left(L^{X}\right)^{E}$ is countably compact according to the parameter $k$, if and only if for every countable subfamily $\mathscr{U} \subseteq \mathscr{C}\left(L^{X}\right)^{E}$, it follows that

$$
\chi_{\mathscr{T}_{k}}(\mathscr{U}) \leq\left[[h \widetilde{\Xi} \bigvee \mathscr{U}] \leq \bigvee_{\mathscr{V} \in 2^{(\mathscr{U})}}[h \widetilde{\Xi} \bigvee \mathscr{V}]\right]
$$

In the light of the similar observations, we may present the parameterized degree of countably compactness in the context of lattice valued $L$-fuzzy soft sets in the following manner. 
Definition 11. Let $\tau: K \rightarrow L^{\left(L^{X}\right)^{E}}$ be a map and $g \in\left(L^{X}\right)^{E}$. Define such a map $\mathscr{C}$ com $_{\tau}: K \rightarrow L^{\left(L^{X}\right)^{E}}$ as follows.

$$
\begin{aligned}
& \mathscr{C} \operatorname{com}_{\tau}(k, g)=\bigwedge_{\mathscr{U} \subseteq \mathscr{C}\left(L^{X}\right)^{E}}\left[\tau_{k}(\mathscr{U}) \leq\left[[g \tilde{\Xi} \bigvee \mathscr{U}] \leq \bigvee_{\mathscr{V} \in 2^{(\mathscr{U})}}[g \widetilde{\Xi} \bigvee \mathscr{V}]\right]\right] \\
& =\bigwedge_{\mathscr{U} \subseteq \mathscr{C}\left(L^{X}\right)^{E}}\left(\tau_{k}(\mathscr{U}) \mapsto\left([g \widetilde{\Xi} \bigvee \mathscr{U}] \mapsto \bigvee_{\mathscr{V} \in 2^{(\mathscr{U})}}[g \widetilde{\sqsubseteq} \bigvee \mathscr{V}]\right)\right) \\
& =\bigwedge_{\mathscr{U} \subseteq \mathscr{C}\left(L^{X}\right)^{E}}\left(\bigwedge_{h \in \mathscr{U}} \tau_{k}(h) \mapsto\left(\bigwedge_{x \in X} \bigwedge_{e \in E}\left(g_{e}^{\prime}(x) \vee \bigvee_{h \in \mathscr{U}} h_{e}(x)\right) \mapsto \bigvee_{\left.\mathscr{V} \in 2^{(\mathscr{U}}\right)} \bigwedge_{x \in X} \bigwedge_{e \in E}\left(g_{e}^{\prime}(x) \vee \bigvee_{h \in \mathscr{V}} h_{e}(x)\right)\right)\right)
\end{aligned}
$$

If $(X, \tau)$ is an L-fuzzy $(E, K)$-soft topological space, then the value $\mathscr{C}$ com $_{\tau}(k, g)$ is called the countably compactness degree of $g$ with respect to the parameter $k$. So $g$ is said to be countably compact L-fuzzy soft set with respect to $k$ if $\mathscr{C} \operatorname{com}_{\tau}(k, g)=1_{L}$. In this manner, the countably compactness degree of $g$ in the whole space $(X, \tau)$ is computed by the value $\mathscr{C} \operatorname{com}_{\tau}(g)=\bigwedge_{k \in K} \mathscr{C} \operatorname{com}_{\tau}(k, g)$. So the $L$-fuzzy soft set $g$ is said to be countably compact in the fuzzy soft space $(X, \tau)$ if $\mathscr{C} \operatorname{com}_{\tau}(g)=1_{L}$. Hence if $\mathscr{C} \operatorname{com}_{\tau}(\widetilde{1})=1_{L}$, then the whole space $(X, \tau)$ is said to be countably compact.

According to the properties of implication operation $\mapsto$, the following lemma can be proved.

Lemma 2. Let $(X, \tau)$ be an $L$-fuzzy $(E, K)$-soft topological space, $g \in\left(L^{X}\right)^{E}$ and $k \in K$. Then $\mathscr{C} \operatorname{com}_{\tau}(k, g) \geq a$ if and only if for each countable subfamily $\mathscr{U} \in \mathscr{C}\left(L^{X}\right)^{E}$,

$$
\tau_{k}(\mathscr{U}) \wedge[g \widetilde{\subseteq} \bigvee \mathscr{U}] \wedge a \leq \bigvee_{\mathscr{V} \in 2^{(\mathscr{U})}}[g \widetilde{\Xi} \bigvee \mathscr{V}]
$$

Theorem 1. Let $(X, \tau)$ be an $L$-fuzzy $(E, K)$-soft topological space, $k \in K$ and $g \in\left(L^{X}\right)^{E}$. Then we can characterize the parameterized countably compactness degree by the following equality.

$$
\mathscr{C} \operatorname{com}_{\tau}(k, g)=\bigvee\left\{a \in L \mid \tau_{k}(\mathscr{U}) \wedge[g \widetilde{\subseteq} \bigvee \mathscr{U}] \wedge a \leq \bigvee_{\mathscr{V} \in 2^{(\mathscr{U})}}[g \widetilde{\subseteq} \bigvee \mathscr{V}], \forall \mathscr{U} \subseteq \mathscr{C}\left(L^{X}\right)^{E}\right\}
$$

Proof. The claim of the theorem is proved via Lemma 2.

According to the following theorem, we may say that each compact $L$-fuzzy soft set is also countably compact.

Theorem 2. Let $(X, \tau)$ be an L-fuzzy $(E, K)$-soft topological space and $g \in\left(L^{X}\right)^{E}$. Then the relation between the parameterized degree of compactness and the parameterized degree of countably compactness of $g$ is as follows

$$
\operatorname{com}_{\tau}(k, g) \leq \mathscr{C} \operatorname{com}_{\tau}(k, g), \quad \text { for all } k \in K .
$$

Proof. It is straightforward by the Definitions 9 and 11, and therefore omitted.

The following result shows that he union of two countably compact $L$-fuzzy soft sets is countably compact too.

Theorem 3. Let $(X, \tau)$ be an $L$-fuzzy $(E, K)$-soft topological space and $g, h \in\left(L^{X}\right)^{E}$. Then the following inequality is satisfied for each $k \in K$,

$$
\mathscr{C} \operatorname{com}_{\tau}(k, g \sqcup h) \geq \mathscr{C} \operatorname{com}_{\tau}(k, g) \wedge \mathscr{C} \operatorname{com}_{\tau}(k, h)
$$


Proof. Let $g, h \in\left(L^{X}\right)^{E}$ and $k \in K$ be given, then

$$
\begin{aligned}
& \mathscr{C} \operatorname{com}_{\tau}(k, g \sqcup h)=\bigvee\left\{a \in L \mid \tau_{k}(\mathscr{U}) \wedge[(g \sqcup h) \widetilde{\Xi} \bigvee \mathscr{U}] \wedge a \leq \bigvee_{\mathscr{V} \in 2_{\mathscr{U}}}[(g \sqcup h) \widetilde{\widetilde{\Xi}} \bigvee \mathscr{V}], \forall \mathscr{U} \subseteq \mathscr{C}\left(L^{X}\right)^{E}\right\} \\
& =\bigvee\left\{a \in L \mid \tau_{k}(\mathscr{U}) \wedge[g \widetilde{\Xi} \bigvee \mathscr{U}] \wedge[h \widetilde{\sqsubseteq} \bigvee \mathscr{U}] \wedge a \leq \bigvee_{\mathscr{V} \in 2^{\mathscr{U}}}[g \widetilde{\sqsubseteq} \bigvee \mathscr{V}] \wedge[h \widetilde{\Xi} \bigvee \mathscr{V}], \forall \mathscr{U} \subseteq \mathscr{C}\left(L^{X}\right)^{E}\right\} \\
& \geq \bigvee\left\{a \in L \mid \tau_{k}(\mathscr{U}) \wedge[g \widetilde{\Xi} \bigvee \mathscr{U}] \wedge a \leq \bigvee_{\mathscr{V} \in 2^{\mathscr{U}}}[g \widetilde{\sqsubseteq} \bigvee \mathscr{V}], \forall \mathscr{U} \subseteq \mathscr{C}\left(L^{X}\right)^{E}\right\} \\
& \wedge \bigvee\left\{a \in L \mid \tau_{k}(\mathscr{U}) \wedge[h \widetilde{\Xi} \bigvee \mathscr{U}] \wedge a \leq \bigvee_{\mathscr{V} \in 2^{\mathscr{U}}}[h \widetilde{\Xi} \bigvee \mathscr{V}], \forall \mathscr{U} \subseteq \mathscr{C}\left(L^{X}\right)^{E}\right\} \\
& =\mathscr{C} \operatorname{com}_{\tau}(k, g) \wedge \mathscr{C} \operatorname{com}_{\tau}(k, h) .
\end{aligned}
$$

Hence this completes the proof.

The following result regards the intersection of a countably compact $L$-fuzzy soft set and a closed $L$-fuzzy soft set, is countably compact.

Theorem 4. Let $(X, \tau)$ be an L-fuzzy $(E, K)$-soft topological space and $g, h \in\left(L^{X}\right)^{E}$. Then the following inequality is valid for each $k \in K$,

$$
\mathscr{C} \operatorname{com}_{\tau}(k, g \sqcap h) \geq \mathscr{C} \operatorname{com}_{\tau}(k, g) \wedge \tau_{k}^{*}(h)
$$

Proof. Let $g, h \in\left(L^{X}\right)^{E}$ and $k \in K$ be given, then

$$
\begin{aligned}
& \mathscr{C} \operatorname{com}_{t} a u(k, g \sqcap h)=\bigvee\left\{a \in L \mid \tau_{k}(\mathscr{U}) \wedge[(g \sqcap h) \widetilde{\sqsubseteq} \bigvee \mathscr{U}] \wedge a \leq \bigvee_{\mathscr{V} \in 2^{\mathscr{U}}}[(g \sqcap h) \widetilde{\sqsubseteq} \bigvee \mathscr{V}], \forall \mathscr{U} \subseteq \mathscr{C}\left(L^{X}\right)^{E}\right\} \\
& =\bigvee\left\{a \in L \mid \tau_{k}(\mathscr{U}) \wedge\left[g \widetilde{\widetilde{\Xi}} h^{\prime} \sqcup \bigvee \mathscr{U}\right] \wedge a \leq \bigvee_{\mathscr{V} \in 2^{\mathscr{U}}}\left[g \widetilde{\sqsubseteq} h^{\prime} \sqcup \bigvee \mathscr{V}\right], \forall \mathscr{U} \subseteq \mathscr{C}\left(L^{X}\right)^{E}\right\} \\
& \geq\left\{a \wedge \tau_{k}^{*}(h) \mid \tau_{k}(\mathscr{U}) \wedge[g \widetilde{\sqsubseteq} \bigvee \mathscr{U}] \wedge q \leq \bigvee_{\mathscr{V} \in 2^{(\mathscr{U})}}[g \widetilde{\sqsubseteq} \bigvee \mathscr{V}], \forall \mathscr{U} \subseteq \mathscr{C}\left(L^{X}\right)^{E}\right\} \\
& \geq \mathscr{C} \operatorname{com}_{\tau}(k, g) \wedge \tau_{k}^{*}(h)
\end{aligned}
$$

Hence this completes the proof.

Corollary 1. Let $(X, \tau)$ be an L-fuzzy $(E, K)$-soft topological space. Then for each $g \in\left(L^{X}\right)^{E}$ the relation between the $k$-parameterized degree of countably compactness of $g$ and the degree of being closed is as follows:

$$
\mathscr{C} \operatorname{com}_{\tau}(k, g) \geq \mathscr{C} \operatorname{com}_{\tau}(k, \tilde{1}) \wedge \tau_{k}^{*}(g)
$$

The following result regards that the property of being countably compactness of an $L$-fuzzy soft set is preserved under continuous $L$-fuzzy soft mapping.

Theorem 5. Let $\varphi_{\psi, \eta}:\left(X_{1}, \tau^{1}\right) \rightarrow\left(X_{2}, \tau^{2}\right)$ be a continuous L-fuzzy soft mapping between L-fuzzy $\left(E_{1}, K_{1}\right)$-soft and L-fuzzy $\left(E_{2}, K_{2}\right)$-soft topological spaces. Then for each $k \in K_{1}$ and $g \in\left(L^{X_{1}}\right)^{E_{1}}$, we have

$$
\mathscr{C} \operatorname{com}_{\tau^{1}}(k, g) \leq \mathscr{C} \operatorname{com}_{\tau^{2}}\left(\eta(k), \varphi_{\psi}(g)\right)
$$


Proof. Let $k \in K_{1}$ and $g$ be a fuzzy soft set on $X_{1}$ with the parameter set $E_{1}$. Then one gets

$$
\begin{aligned}
\mathscr{C} \operatorname{com}_{\tau^{2}}\left(\eta(k), \varphi_{\psi}(g)\right) & =\bigvee\left\{a \in L \mid \tau_{\eta(k)}^{2}(\mathscr{U}) \wedge\left[\varphi_{\psi}(g) \widetilde{\subseteq} \bigvee \mathscr{U}\right] \wedge a \leq \bigvee_{\mathscr{V} \in 2^{\mathscr{U}}}\left[\varphi_{\psi}(g) \widetilde{\subseteq} \bigvee \mathscr{V}\right], \forall \mathscr{U} \subseteq \mathscr{C}\left(L^{X_{2}}\right)^{E_{2}}\right\} \\
& \geq \bigvee\left\{a \mid\left(\tau^{1}\right)_{k}\left(\varphi_{\psi}^{-1}(\mathscr{U})\right) \wedge\left[g \widetilde{\subseteq} \bigvee \varphi_{\psi}^{-1}(\mathscr{U})\right] \wedge a \leq \bigvee_{\mathscr{V} \in 2_{\mathscr{\mathscr { U }}}}\left[g \widetilde{\subseteq} \bigvee \varphi_{\psi}^{-1}(\mathscr{V})\right], \forall \mathscr{U} \subseteq \mathscr{C}\left(L^{X_{2}}\right)^{E_{2}}\right\} \\
& \geq \bigvee\left\{a \in L \mid \tau_{k}^{1}(\mathscr{P}) \wedge[g \widetilde{\subseteq} \bigvee \mathscr{P}] \wedge a \leq \bigvee_{\mathscr{R} \in 2^{\mathscr{P}}}[g \subseteq \widetilde{\complement} \bigvee \mathscr{R}], \forall \mathscr{P} \in \mathscr{C}\left(L^{X_{1}}\right)^{E_{1}}\right\} \\
& =\mathscr{C} \operatorname{com}_{\tau^{1}}(k, g) .
\end{aligned}
$$

Hence the proof is completed as desired.

\section{Parameterized Degree of the Lindelöf Property}

In this section, we gain the extension of the parameterized degree of Lindelöf property with its fundamental properties.

Definition 12. Let $\mathscr{T}=\left\{\mathscr{T}_{k}\right\}_{k \in K}$ be an $(E, K)$-soft L-topology on $X$ and $h \in\left(L^{X}\right)^{E}$. The L-fuzzy soft set $h$ is said to have the Lindelöf property in $(X, \mathscr{T})$, if for every $k$ and every open $k$-cover $\mathscr{U} \subseteq \mathscr{T}_{k}$ of $h$ there exists a countable subfamily $\mathscr{V} \subseteq \mathscr{U}$ which covers $h$, i.e.,

$$
[h \widetilde{\Xi} \bigvee \mathscr{U}] \leq \bigvee_{\mathscr{V} \in 2^{|\mathscr{U}|}}[h \widetilde{\Xi} \bigvee \mathscr{V}]
$$

This implies that

$$
\left[[h \widetilde{\subseteq} \bigvee \mathscr{U}] \leq \bigvee_{\mathscr{V} \in 2^{|\mathscr{U}|}}[h \widetilde{\Xi} \bigvee \mathscr{V}]\right]=1_{L}, \text { for any } \mathscr{U} \subseteq \mathscr{T}_{k}
$$

Hence we may infer that $h \in\left(L^{X}\right)^{E}$ has the Lindelöf property according to the parameter $k$, if and only if for every subfamily $\mathscr{U} \subseteq\left(L^{X}\right)^{E}$, it follows that

$$
\left.\chi_{\mathscr{T}_{k}}(\mathscr{U}) \leq[h \tilde{\subseteq} \bigvee \mathscr{U}] \leq \bigvee_{\mathscr{V} \in 2^{|\mathscr{U}|}}[h \widetilde{\subseteq} \bigvee \mathscr{V}]\right]
$$

In the light of the similar observations, we may present the parameterized degree of having Lindelöf property in the context of lattice valued $L$-fuzzy soft sets as follows.

Definition 13. Let $\tau: K \rightarrow L^{\left(L^{X}\right)^{E}}$ be a map and $g \in\left(L^{X}\right)^{E}$. Define such a map Lip $\tau: K \rightarrow L^{\left(L^{X}\right)^{E}}$ as follows.

$$
\begin{aligned}
\operatorname{Lip}_{\tau}(k, g) & =\bigwedge_{\mathscr{U} \subseteq\left(L^{X}\right)^{E}}\left[\tau_{k}(\mathscr{U}) \leq\left[[g \widetilde{\subseteq} \bigvee \mathscr{U}] \leq \bigvee_{\mathscr{V} \in 2^{|\mathscr{U}|}}[g \widetilde{\subseteq} \bigvee \mathscr{V}]\right]\right] \\
& =\bigwedge_{\mathscr{U} \subseteq\left(L^{X}\right)^{E}}\left(\tau_{k}(\mathscr{U}) \mapsto\left([g \widetilde{\subseteq} \bigvee \mathscr{U}] \mapsto \bigvee_{\mathscr{V} \in 2^{|\mathscr{U}|}}[g \widetilde{\subseteq} \bigvee \mathscr{V}]\right)\right) \\
& =\bigwedge_{\mathscr{U} \subseteq\left(L^{X}\right)^{E}}\left(\bigwedge_{h \in \mathscr{U}} \tau_{k}(h) \mapsto\left(\bigwedge_{x \in X} \bigwedge_{e \in E}\left(g_{e}^{\prime}(x) \vee \bigvee_{h \in \mathscr{U}} h_{e}(x)\right) \mapsto \bigvee_{\mathscr{V} \in 2^{|\mathscr{U}|} \mid x \in X} \bigwedge_{e \in E}\left(g_{e}^{\prime}(x) \vee \bigvee_{h \in \mathscr{V}} h_{e}(x)\right)\right)\right)
\end{aligned}
$$

If $(X, \tau)$ is an L-fuzzy $(E, K)$-soft topological space, then the value Lip $\tau(k, g)$ is called the parameterized degree of having Lindelöf property of $g$ with respect to $k$ in $(X, \tau)$. So $g$ is said to have Lindelöf property with respect to $k$ if $L_{i p}(k, g)=1_{L}$. In this manner, the Lindelöfness degree of $g$ in the whole space $(X, \tau)$ is computed by the value $\operatorname{Lip}_{\tau}(g)=\bigwedge_{k \in K} \operatorname{Lip}_{\tau}(k, g)$. So the L-fuzzy soft set $g$ is said to has Lindelöf property in the fuzzy soft space $(X, \tau)$ if Lip $\tau(g)=1_{L}$. Hence if Lip $\tau(\widetilde{1})=1_{L}$, then the whole space $(X, \tau)$ is said to be Lindelöf. 
Analogous to the parameterized degree of the countably compactness, we gain the following results.

Lemma 3. Let $(X, \tau)$ be an $L$-fuzzy $(E, K)$-soft topological space, $g \in\left(L^{X}\right)^{E}$ and $k \in K$. Then Lip $\tau(k, g) \geq a$ if and only if for any $\mathscr{U} \in\left(L^{X}\right)^{E}$,

$$
\tau_{k}(\mathscr{U}) \wedge[g \widetilde{\sqsubseteq} \bigvee \mathscr{U}] \wedge a \leq \bigvee_{\mathscr{V} \in 2^{|\mathscr{U}|}}[g \sqsubseteq \widetilde{\sqsubseteq} \bigvee \mathscr{V}]
$$

Theorem 6. Let $(X, \tau)$ be an L-fuzzy $(E, K)$-soft topological space, $k \in K$ and $g \in\left(L^{X}\right)^{E}$. Then we can characterize the having parameterized degree of Lindelöfness by the following equality.

$$
\operatorname{Lip}_{\tau}(k, g)=\bigvee\left\{a \in L \mid \tau_{k}(\mathscr{U}) \wedge[g \widetilde{\sqsubseteq} \bigvee \mathscr{U}] \wedge a \leq \bigvee_{\mathscr{V} \in 2^{|\mathscr{U}|}}[g \widetilde{\sqsubseteq} \bigvee \mathscr{V}], \forall \mathscr{U} \subseteq\left(L^{X}\right)^{E}\right\}
$$

Theorem 7. Let $(X, \tau)$ be an L-fuzzy $(E, K)$-soft topological space and $g, h \in\left(L^{X}\right)^{E}$. Then the following inequality is satisfied for each $k \in K$,

$$
\operatorname{Lip}_{\tau}(k, g \sqcup h) \geq \operatorname{Lip}_{\tau}(k, g) \wedge \operatorname{Lip}_{\tau}(k, h)
$$

Theorem 8. Let $(X, \tau)$ be an L-fuzzy $(E, K)$-soft topological space and $g, h \in\left(L^{X}\right)^{E}$. Then the following inequality is valid for each $k \in K$,

$$
\operatorname{Lip}_{\tau}(k, g \sqcap h) \geq \operatorname{Lip}_{\tau}(k, g) \wedge \tau_{k}^{*}(h)
$$

Corollary 2. Let $(X, \tau)$ be an L-fuzzy $(E, K)$-soft topological space. Then for each $g \in\left(L^{X}\right)^{E}$ the relation between the $k$-parameterized degree of having Lindelöf property of $g$ and the degree of being closed is as follows:

$$
\operatorname{Lip}_{\tau}(k, g) \geq \operatorname{Lip}_{\tau}(k, \widetilde{1}) \wedge \tau_{k}^{*}(g)
$$

The following result shows that an $L$-fuzzy soft set which is both countable compact and has Lindelöf property is compact.

Theorem 9. Let $(X, \tau)$ be an L-fuzzy $(E, K)$-soft topological space and $g \in\left(L^{X}\right)^{E}$. Then the relation among the parameterized degree of beng compact, countably compact and Lindelöfness is as follows

$$
\operatorname{Lip}_{\tau}(k, g) \wedge \mathscr{C} \operatorname{com}_{\tau}(k, g) \leq \operatorname{com}_{\tau}(k, g), \quad \text { for any } k \in K
$$

Proof. Let $a \in c(L)$ such that $a \leq \operatorname{Lip}_{\tau}(k, g) \wedge \mathscr{C} \operatorname{com}_{\tau}(k, g)$ be satisfied for any $k \in K$. Suppose for any $\mathscr{U} \in\left(L^{X}\right)^{E}$, since $a \leq \operatorname{Lip}_{\tau}(k, g)$ and $a \leq \mathscr{C} \operatorname{com}_{\tau}(k, g)$, we have that $\tau_{k}(\mathscr{U}) \wedge[g \widetilde{\Xi} \bigvee \mathscr{U}] \wedge a \leq \bigvee_{\mathscr{V} \in 2^{|\mathscr{U}|}}[g \widetilde{\widetilde{\complement}} \bigvee \mathscr{V}]$, by Lemma 3. Since $\mathscr{V} \in 2^{|\mathscr{U}|}$, that is $\mathscr{V} \in \mathscr{C}\left(L^{X}\right)^{E}$, then $\tau_{k}(\mathscr{U}) \leq \tau_{k}(\mathscr{V})$ and hence, $\tau_{k}(\mathscr{V}) \wedge[g \widetilde{\widetilde{\complement}} \bigvee \mathscr{V}] \wedge a \leq \bigvee_{\mathscr{W} \in 2^{(\mathscr{V})}}[g \subseteq \widetilde{\complement} \bigvee \mathscr{W}]$ by Lemma 2 Further we get the following

$$
\begin{aligned}
\tau_{k}(\mathscr{U}) \wedge[g \widetilde{\Xi} \bigvee \mathscr{U}] \wedge a & \leq \tau_{k}(\mathscr{U}) \wedge \bigvee_{\mathscr{V} \in 2^{|\mathscr{U}|}}[g \widetilde{\sqsubseteq} \bigvee \mathscr{V}] \wedge a \leq \bigvee_{\mathscr{V} \in 2^{|\mathscr{U}|}}\left(\tau_{k}(\mathscr{V}) \wedge[g \widetilde{\sqsubseteq} \bigvee \mathscr{V}] \wedge a\right) \\
& \leq \bigvee_{\mathscr{V} \in 2^{|\mathscr{U}|}}\left(\bigvee_{\mathscr{W} \in 2^{(\mathscr{V})}}[g \widetilde{\sqsubseteq} \bigvee \mathscr{W}]\right) \leq \bigvee_{\mathscr{W} \in 2^{(\mathscr{U})}}[g \widetilde{\sqsubseteq} \bigvee \mathscr{W}]=\bigvee_{\mathscr{V} \in 2^{(\mathscr{U})}}[g \widetilde{\sqsubseteq} \bigvee \mathscr{V}] .
\end{aligned}
$$

This inequality implies the fact that $\left(\tau_{k}(\mathscr{U}) \wedge a\right) \leq\left([g \widetilde{\Xi} \bigvee \mathscr{U}] \mapsto \bigvee_{\mathscr{V} \in 2^{(\mathscr{U})}}[g \widetilde{\complement} \bigvee \mathscr{V}]\right)$, for any $\mathscr{U} \subseteq\left(L^{X}\right)^{E}$. By the implication operation properties, we may conclude that $a \leq \tau_{k}(\mathscr{U}) \mapsto\left([g \widetilde{\sqsubseteq} \bigvee \mathscr{U}] \mapsto \bigvee_{\mathscr{V} \in 2^{(\mathscr{U})}}[g \widetilde{\sqsubseteq} \bigvee \mathscr{V}]\right)$, for any $\mathscr{U} \subseteq\left(L^{X}\right)^{E}$. This completes the proof as desired. 


\section{Conclusion}

In the present paper, the parameterized extensions of the countably compactness and the Lindelöf property have been identified and investigated. Hence, we suitably transferred most useful compactness types to the graded parameterized universe named fuzzy soft space. We are hopeful that our theoretical investigations will be helpful for the applied researches.

\section{Competing interests}

The authors declare that they have no competing interests.

\section{Authors' contributions}

All authors have contributed to all parts of the article. All authors read and approved the final manuscript.

\section{References}

[1] T.M. Al-shami, Lj.D.R. Kocinac, The equivalence between the enriched and extended soft topologies, Appl. Comput. Math. 28(2) (2019) 149-162.

[2] T. M. Al-shami, M. E. El-Shafei, On soft compact and soft Lindelöf spaces via soft pre-open sets, Annals of Fuzzy Mathematics and Informatics 17(1) (2019) 79-100.

[3] T.M. Al-shami, MA.. Al-Shumrani, B.A. Asaad, Some generalized forms of soft compactness and soft Lindelöfness via soft $\alpha$ open sets, Italian Journal of Pure and Applied Mathematics 41 (2020) 680-704.

[4] T. M. Al-shami, M. E. El-Shafei and M. Abo-Elhamayel, Almost soft compact and approximately soft Lindelöf spaces, J. Taibah Univ. Sci. 12 (5) (2018) 620-630.

[5] T. M. Al-shami, M. E. El-Shafei, On soft compact and soft Lindelöf spaces via soft pre-open sets, Ann. Fuzzy Math. Inform. 17 (1) (2019) 79-100.

[6] T. M. Al-shami, M. E. El-Shafei, Partial belong relation on soft separation axioms and decision making problem: two birds with one stone Soft Comput. 24 (2020) 5377-5387.

[7] B. Ahmad, A. Kharal, On fuzzy soft sets, Adv. Fuzzy Syst. 2009 (2009) Article ID 586507.

[8] J. C. R. Alcantud, T. J. Mathew, Separable fuzzy soft sets and decision making with positive and negative attributes, Appl. Soft Comput. 59 (2017) 586-595.

[9] A. Aygünoğlu, V. Çetkin, H. Aygün, An introduction to fuzzy soft topological spaces, Hacet. J. Math. Stat. 43(2) (2014) 197-208.

[10] C. L. Chang, Fuzzy topological spaces, J.Math. Anal. Appl. 24 (1968) 182-190.

[11] V. Çetkin, H. Aygün, A note on fuzzy soft topological spaces, The 8th Congerence of the European Society for Fuzzy Logic and Technology, Atlantis Press Proceedings, EUSFLAT 2013, doi:10.2991/eusflat.2013.8, 56-60, Milan- Italy, (2013).

[12] V. Çetkin, A. Aygünoğlu, H. Aygün, A topological view on application of L-fuzzy soft sets: Compactness, J. Intell. Fuzzy Syst. 32 (2017) 781-790.

[13] V. Çetkin, H. Aygün, A topological view on L-fuzzy soft sets: Connectedness degree, J. Intell. Fuzzy Syst. 34 (2018) 1975-1983.

[14] V. Çetkin, Parameterized degree of semi-precompactness in the fuzzy soft universe, J. Intell. Fuzzy Syst. 36 (2019) 3661-3670.

[15] V. Çetkin, On measures of parameterized fuzzy compactness, Filomat, 34 (9) (2020) 2927-2938.

[16] M. E. El-Shafei, M. Abo-Elhamayel and T. M. Al-shami, Partial soft separation axioms and soft compact spaces, Filomat 32 (13) (2018) 4755-4771.

[17] M. E. El-Shafei, T. M. Al-shami, Applications of partial belong and total non-belong relations on soft separation axioms and decision-making problem, Comp. Appl. Math. 39 (138) (2020). https://doi.org/10.1007/s40314-020-01161-3.

[18] G. Gierz et al., A compendium of continuous lattices, Springer-Verlag, 1980. 
[19] A. Kharal, B. Ahmad, Mappings on fuzzy soft classes, Adv. Fuzzy Syst. 2009 (2009) Article ID 407890.

[20] H.Y. Li, F.G. Shi, Measures of fuzzy compactness in L-fuzzy topological spaces, Comput. Math. Appl. 59 (2) (2010) $941-947$.

[21] R. Lowen, Fuzzy topological spaces and fuzzy compactness, J.Math. Anal. Appl. 56 (1976) 621-633.

[22] X. Ma, J. Zhan, M.I. Ali, N. Mehmood, A survey of decision making methods based on two classes of hybrid soft set models, Artificial Intelligence Review 49(4) (2018) 511-529.

[23] P. K. Maji, R. Biswas, A.R. Roy, Fuzzy soft sets, J. Fuzzy Math. 9(3) (2001) 589-602.

[24] D. Molodtsov, Soft set theory-First results, Comput. Math. Appl. 37 (4/5) (1999) 19-31.

[25] W. Pan, J. Zhan, Two novel products of IFP-intuitionistic fuzzy soft sets and corresponding decision making methods, Journal of Discrete Mathematical Sciences and Cryptography 21 (3) (2018) 631-646.

[26] X.D.Peng, Y. Yang, Algorithms for interval-valued fuzzy soft sets in stochastic multi- criteria decision making based on regret theory and prospect theory with combined weight, Appl. Soft Comput. 54 (2017) 415-430.

[27] G. N. Raney, A subdirect-union representation for completely distributive complete lattices, Proc. Amer. Math. Soc. 4 (1953) 518-522.

[28] A. R. Roy, P. K. Maji, A fuzzy soft set theoretic approach to decision making problems, J. Comput. Appl. Math. 203 (2007) 412-418.

[29] F.-G. Shi, A new notion of fuzzy compactness in L-topological spaces, Inf. Sci. 173 (2005) 35-48.

[30] F.-G. Shi, A new definition of fuzzy compactness, Fuzzy Sets Syst. 158 (2007) 1486-1495.

[31] F.-G. Shi, Countably compactness and the Lindelöf property of L-fuzzy sets, Iran. J. Fuzzy Syst. 1 (1) (2004) 75-84.

[32] A. P. Sostak, Two decades of fuzzy topology: Basic ideas, notions and results, Russ. Math. Surveys 44 (1989) 1-23.

[33] J. Zhan, Q. Wang, Certain types of soft coverings based rough sets with applications, Int. J. Mach. Learn. Cybern. (2018) doi/10.1007/s13042-018-0785-x.

[34] L. Zhang, J. Zhan, J. C. R. Alcantud Novel classes of fuzzy soft $\beta$-coverings-based fuzzy rough sets with applications to multicriteria fuzzy group decision making, Soft Comput. (2018) https://doi.org/10.1007/s00500-018-3470-9. 